\title{
ADJUSTMENT OF THE MECHANICAL PROPERTIES OF SILICONE FOR APPLICATIONS IN THE MIDDLE EAR
}

\author{
Tanja Heemeier ${ }^{1}$, Tammo Lüßenhop ${ }^{1}$, Peter Behrens ${ }^{1}$ \\ ${ }^{1}$ Institut für Anorganische Chemie, Leibniz Universität Hannover, Callinstraße 9, D-30167 Hannover, Germany \\ Tanja.Heemeier@acb.uni-hannover.de
}

\begin{abstract}
In case of loss of the auditory ossicular chain, the application of a middle ear prosthesis is necessary. For this prosthesis, a silicone pad shall be developed to minimize pressure peaks and for the prevention of forcing of the tympanic membrane. It is important to adjust the mechanical properties for best hearing quality. Therefore different siloxanes and additives like nanoporous silica nanoparticles are used. The latter also can act as drug carriers. The visco-elastic properties of the silicone can be changed significantly by slight variations of the composition.
\end{abstract}

Keywords: ear-drum pad, silicone, nanoporous silica nanoparticles, drug carrier

\section{Introduction}

A possible complication in the application of a middle ear prosthesis is the movement of the prosthesis through the tympanic membrane. To prevent this complication, usually a piece of cartilage is placed between the headpiece of the prosthesis and the tympanic membrane. As a synthetic substitute, we aim to develop a silicone pad made of poly(dimethylsiloxane) (PDMS). For this purpose, the mechanical properties of the silicone have to be adjusted in order to minimize pressure peaks on the ear-drum; on the other hand, an impairment of sound transmission has to be avoided. Furthermore nanoporous silica nanoparticles (NPSNPs) shall be embedded as a filler and a possible drug carrier.

\section{Methods}

Mechanical properties of PDMS are adjusted by adding different siloxanes, NPSNPs and further additives. The NPSNPs have to be functionalized for embedding them into the silicone. Characterization of the silicone samples has been carried out by rheology measurements (Fig. 1). Examination of the release behavior from nanoporous silica nanoparticles will also be performed.

\section{Results}

By adding siloxanes to the silicone mixture it is possible to regulate the mechanical properties of PDMS. The rigidity of the silicone can be increased by the addition of low-viscosity siloxanes and NPSNPs whereas high-viscosity siloxanes lead to increased flexibility. It was observed that slight variation in cross-linking causes significant changes in the visco-elastic properties (Fig. 1).

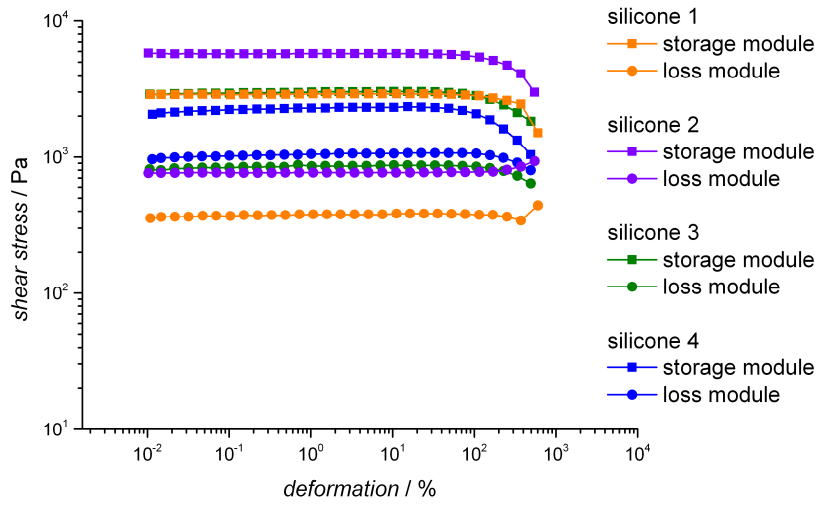

Figure 1: Varying the composition of the silicone shows different visco-elastic properties. Silicone 1: vinyl-PDMS (970 mPas) + 28\% H-PDMS (12 mPas); silicone 2: vinylPDMS (4700 mPas) + 17\% H-PDMS (12 mPas); silicone 3: vinyl-PDMS (4700 mPas) + 25\% H-PDMS (12 mPas); silicone 4: vinyl-PDMS (4700 mPas) + 30\% H-PDMS (29 mPas) (vinyl-PDMS: vinyl-terminated PDMS, H-PDMS: partially substituted methyl groups by hydrogen).

\section{Discussion}

Application of silicones as ear-drum pad could be a promising alternative for the currently used cartilage discs. Moreover, incorporated NPSNPs offer the possibility for a drug release which could promote healing.

\section{Acknowledgement}

This work was supported by the DFG within the Collaborative Research Program SFB 599 "Sustainable bioresorbable and permanent implants based on metallic and ceramic materials" (TP D1). 